Presented at: VII Workshop on "Advanced

Accelerator Concepts

CONF-9610210- 4

Lake Tahoe, CA

October 13-18, 1996

BNL- 63635

\title{
Status of the BNL IFEL Accelerator
}

A. van Steenbergen ${ }^{1}$ J. Gallardo ${ }^{1}$, J. Sandweiss ${ }^{2}$, M. Babzien ${ }^{1}$, J.-M. Fang ${ }^{3}$, K. Kusche ${ }^{1}$, R. Malone ${ }^{1}$, I. Pogorelsky ${ }^{1}$, X. Qiu ${ }^{4}$, T. Romano ${ }^{1}$, J. Sheehan ${ }^{1}$, J. Skaritka ${ }^{1}$, X.-J. Wang ${ }^{1}$

${ }^{1}$ Brookhaven National Laboratory, Upton, NY 11973

${ }^{2}$ Physics Department, Yale University, New Haven, CT 06511

${ }^{3}$ Department of Applied Physics, Columbia U., NY 10027

${ }^{4}$ Physics Department, State University of New York, Stony Brook, NY 11794

0502395

OSTI

October 1996

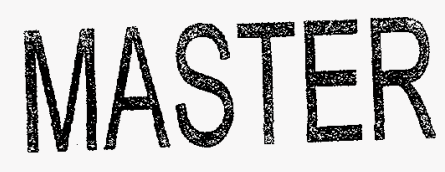

National Synchrotron Light Source

Brookhaven National Laboratory

Upton, NY 11973

Work performed under the auspices of the U.S. Department of Energy, under contract DE-AC02-76CH00016 


\section{DISCLAIMER}

This report was prepared as an account of work sponsored by an agency of the United States Government. Neither the United States Government nor any agency thereof, nor any of their employees, makes any warranty, express or implied, or assumes any legal liability or responsibility for the accuracy, completeness, or usefulness of any information, apparatus, product, or process disclosed, or represents that its use would not infringe privately owned rights. Reference herein to any specific commercial product, process, or service by trade name, trademark, manufacturer, or otherwise does not necessarily constitute or imply its endorsement, recommendation, or favoring by the United States Government or any agency thereof. The views and opinions of authors expressed herein do not necessarily state or reflect those of the United States Government or any agency thereof. 


\section{DISCLAMMER}

Portions of this document may be illegible in electronic image products. Images are produced from the best available original document. 


\title{
Status of the BNL IFEL Accelerator
}

\author{
A.van Steenbergen ${ }^{1}$, J.Gallardo ${ }^{1}$, J.Sandweiss ${ }^{2}$, M.Babzien $^{1}$, J-M Fang ${ }^{3}$, K.Kusche ${ }^{1}$, \\ R.Malone ${ }^{1}$, I.Pogorelsky ${ }^{1}$, X.Qiu ${ }^{4}$, T.Romano ${ }^{1}$, J.Sheehan ${ }^{1}$, J.Skaritka ${ }^{1}$, X-J Wang ${ }^{1}$. \\ 'Brookhaven National Laboratory, Upton, NY 11973 \\ ${ }^{2}$ Physics Department, Yale University, New Haven, CT 06511 \\ ${ }^{3}$ Department of Applied Physics, Columbia U., New York, NY 10027
}

\section{ABSTRACT}

A $40 \mathrm{MeV}$ electron beam, using the inverse free-electron laser interaction, has been accelerated by $\triangle E / E=2.5 \%$ over a distance of $0.47 \mathrm{~m}$. The electrons interact with a $1-2 \mathrm{GW} \mathrm{CO}$ laser beam bounded by a $2.8 \mathrm{~mm}$ ID sapphire circular waveguide in the presence of a tapered wiggler with $B \max \approx 1 \mathrm{~T}$ and a period $2.89 \mathrm{~cm} \leq \lambda_{w} \leq 3.14 \mathrm{~cm}$. The experimental results of $\triangle E / E$ as a function of electron energy $E$, peak magnetic field $B W$ and laser power $W_{1}$ compare well with analytical and 1-D numerical simulations and permit scaling to higher laser power and electron energy. The present status of the IFEL accelerator and planned near term development are indicated.

\section{INTRODUCTION}

The study of the Inverse-Free Electron-Laser (IFEL) as a potential mode of electron acceleration has been pursued at Brookhaven National Laboratory (BNL) for a number of years [1-4]. Recent studies have focused on the development of a low energy, high gradient, IFEL accelerator [5] as a first step toward a multi-module electron accelerator of maximum operating energy of a few GeV. Experimental verification of the IFEL accelerator concept was obtained in 1992 [6], using a radiation wave length of $\lambda=1.65 \mathrm{~mm}$, and more recently [7] using a wavelength of $10.6 \mu \mathrm{m}$. In this report further experimental evidence of the IFEL interaction $(\lambda=10.6 \mu \mathrm{m})$ is presented. The experiment used a $50 \mathrm{MeV}$ electron beam, a $1-$ $5 \mathrm{GW} \mathrm{CO} 2$ laser beam provided by BNL's Accelerator Test Facility (ATF) and a uniquely designed period length tapered wiggler.

The wiggler is a fast excitation electromagnet with stackable, geometrically and magnetically alternating substacks of Vanadium Permendur (VaP) ferromagnetic laminations, periodically interspersed with conductive ( $\mathrm{Cu}$ ), nonmagnetic laminations, which act as eddy current induced field reflectors $[8,9]$. Four current conducting rods, parallel to the wiggler axis, are connected at the ends of the assembly, constituting the excitation loop that drives the wiggler. The overall wiggler stack is easily assembled, is compressed by simple tie rods, and readily permits wiggler period $\left(\lambda_{w}\right)$ variation. Configured as a constant period wiggler, $\lambda_{w}=3.75 \mathrm{~cm}$ and $B_{\max }=1 \mathrm{~T}$, the system has shown [10] an ms pole-to-pole field variation of approximately $0.2 \%$.

The $\mathrm{CO}_{2}$ laser beam is brought into the IFEL interaction region by a low loss dielectric $\left(\mathrm{Al}_{2} \mathrm{O}_{3}\right.$, sapphire) circular waveguide which evidenced very good transmission properties [11] of the high power $\mathrm{CO}_{2}$ laser beam. Extensive studies were carried out to establish optimum coupling into the guide and to measure the transmission loss of the long $(1.0 \mathrm{~m})$ extruded single crystal sapphire guides. Also, because of the overmoded guide configuration (ID $=2.8 \mathrm{~mm}$ ), attempts were made to determine the transverse mode spectrum. To this end various wave guide configurations were tested at low laser beam power with the beam focused to a Gaussian waist with adjustable radius at the entrance of the waveguide. The beam profile was measured using a pyroelectric vidicon TV camera combined with digital frame grabber. For the $2.8 \mathrm{~mm}$. ID sapphire dielectric guide a laser power attenuation factor of $0.2 \mathrm{~dB} / \mathrm{m}$ was measured. The laser beam profile within the guide was inferred by measuring the beam diameter at the guide exit for various guide lengths. The results show that, commensurate with the near constant beam profile within the guide, the mode structure is dominated by the guide fundamental mode only. This is in accord with the absence of mode mixing reported in Ref.[11] for filamentary sapphire guides for $\mathrm{CO}_{2}$ laser radiation transport.

The laser power must be efficiently coupled into the desired mode $\left(\mathrm{H}_{11}\right)$. To determine the transition region over which the mode becomes established, a series of scalar diffraction calculations were performed to find the fields propagating from the coupling aperture. It was found that the mode pattern transformed from the input Gaussian to a stable field distribution over a distance comparable to $Z_{R}$, the Rayleigh length. For the waist sizes employed here, after the mode has stabilized, the amplitude typically fluctuates by $+5 \%$ and the phase by +-0.05 radians. These calculations suggest a $90 \%$ coupling efficiency into the desired mode, consistent with the experimental observations reported below. 
In the IFEL accelerator, the electron beam is accelerated by the interaction with the laser radiation wave in the medium of a periodic wiggler field. The theoretical description of the interaction has been given by a number of authors $[3,12]$. Approximate analytical expressions derived in Ref.[3] were used to parameterize a single acceleration stage. Subsequently, 1-D and 3-D simulation programs were written solving the self consistent system of Lorentz equations for the electrons and the wave equations for the input laser field as discussed in Ref. [12]. The 1-D program has been used to determine the selfconsistent wiggler period length and its taper for given values of electron beam energy and laser power and to calculate the bucket acceptance and bucket leakage for a single or multi module accelerator. The 3-D code has been used to study beam walk-off, transverse phase space distributions and emittance growth.

\section{EXPERIMENTAL ARRANGEMENT and RESULTS}

Extensive IFEL simulation studies were carried out both for a single IFEL accelerator module and for a sequence of IFEL modules. The objective of the present experiment was a proof of principle performance of a single IFEL unit incorporated in beam line II of the ATF $[13,14]$. A schematic layout, specific to the IFEL experiment only, is shown in Fig.1. Beam transport downstream from the nominal $50 \mathrm{MeV}$ Linac is so dimensioned as to yield a dispersion free IFEL interaction region. The electron beam, at the IFEL location, is matched vertically to the natural wiggler betatron amplitude $\beta_{y}=0.17 \mathrm{~m}$, $\alpha_{x}=0.0$ and to a horizontal amplitude $\beta_{x}=0.3 \mathrm{~m}, \alpha_{x}=0.0$. Downstream of the IFEL interaction region the optical system is configured as a momentum spectrometer with adjustable dispersion magnitude $\left(0.0<\eta_{\mathrm{p}}<3.0 \mathrm{~m}\right)$ at a diagnostic endstation; there the beam momentum dispersion is measured by means of a phosphor screen-vidicon TV camera-Spiracon frame grabber. Also shown schematically in Fig. 1 is the $\mathrm{CO}_{2}$ laser beam entry into the interaction region vacuum envelope through a $\mathrm{ZnSe}$ window, and its propagation as a free-space mode, to the sapphire dielectric waveguide entry. With deliberation, the dielectric guide was taken to be $0.6 \mathrm{~m}$ in length, whereas the accelerator module length (wiggler length) was set at $0.47 \mathrm{~m}$. This was done to approximate a mode matching section, enhancing thereby the mode purity in the IFEL module proper.

The design parameters used in this FEL accelerator experiment are listed in Table 1. With optimized overlap of the electron and $\mathrm{CO} 2$ laser beams, both spatially and timewise, and the interleaving of the lower repetition rate $\mathrm{CO}_{2}$ laser pulses with the higher repetition rate electron beam pulses, the IFEL electron beam acceleration could readily be established. Electron acceleration was measured with the spectrometer at the diagnostic screen. An example of the momentum spectrum of the unaccelerated and accelerated electrons is given Fig.2, where the beam intensity distribution is shown versus $\sqrt{ } \beta_{x} \varepsilon_{x}+$ $\eta_{p} \Delta p / p$, with the spectrometer optics adjusted so that $\eta_{p} \Delta p / p \gg \sqrt{ } \beta_{x} \varepsilon_{x}$.

Optimization of the IFEL effect and exploration of parameter space, with variation of the electron beam injection energy, $\mathrm{CO}_{2}$ laser power and wiggler maximum magnetic field magnitude was carried out in several consecutive runs, the results of which established the unambiguous signature of the IFEL acceleration. This is illustrated in Figs.3, 4 and 5 where $(\Delta E / E)_{\text {FEI }}$ is shown both as given by the 1-D model simulations and as obtained experimentally. Fig. 3 shows the relative energy gain for $B_{w}$ and $W_{1}$ constant; in Fig.4 the plot $(\triangle E / E)_{\text {FEL }}$ vs. $B_{w}$ is given and in Fig.5 the relative energy gain versus laser power $W_{1}$ is plotted.

The approximate IFEL design equations [3] are:

$\mathrm{d} \gamma / \mathrm{dz}=\mathrm{A}(\mathrm{K} / \gamma) \mathrm{f}(\mathrm{K}) \sin \psi$ where $\psi=\left(\mathrm{k}+\mathrm{k}_{\mathrm{w}}\right) \mathrm{z}-\mathrm{kct}$

where the normalized laser electric field is $A=\left(e / \mathrm{mc}^{2}\right)\left(1 / \mathrm{R}_{0}\right)\left(\pi \mathrm{W}_{\mathrm{L}} \mathrm{Z}_{\mathrm{o}}\right)^{1 / 2}, \mathrm{~K}=\left(\mathrm{eB} \mathrm{w}_{\mathrm{w}} \lambda_{w}\right) /(2 \pi \mathrm{mc}) \approx 2.7$ is the wiggler parameter, $f(K)=0.38$ is a correction factor due to the linear polarization of the wiggler, $Z_{0}=377 \Omega, R_{0}$ is the waveguide radius and $k$, $\mathrm{k}_{\mathrm{w}}$ are the radiation and wiggler wavevectors, respectively. The resonance condition leads to:

$\lambda=0.5 \lambda_{w} / \gamma^{2}\left(1+K^{2} / 2\right)$

The relative energy gain of the electron beam in a wiggler of length $L_{w}$ is:

$\Delta \gamma / \gamma=(\Delta \mathrm{p} / \mathrm{p})_{\text {IFEL }}=\mathrm{A}\left(\mathrm{K} / \gamma^{2}\right) \mathrm{f}(\mathrm{K}) \sin \psi_{\mathrm{I}} \mathrm{L}_{\mathrm{w}} \quad$ where

$\psi_{r}$ is the resonance phase $\left(45^{\circ}\right.$ for optimum bucket size).

In Fig. 3 the solid line shows the results of the numerical simulations with laser power $W_{1}=1 \mathrm{GW}$ and $B_{w}=10 \mathrm{kG}$ normalized to the maximum experimental value. The agreement of the simulations with the experimental results are good. Similarly, in Fig. 4 experimental results are compared with the simulations for $35 \mathrm{MeV}$ and $40 \mathrm{MeV}$, in both cases the agreement is good. The maximum $(\Delta \mathrm{p} / \mathrm{p})_{\text {FEE }}$ for initial electron energy of $35 \mathrm{MeV}$ leads to a value of the magnetic field $B_{w}=$ $8.35 \mathrm{kG}$, to be compared with the experimental value of $8.44 \mathrm{kG}$, and for $E=40 \mathrm{MeV}$, the calculated $B_{w}$ is $9.98 \mathrm{kG}$ and the 
experimental value was $B_{w}=9.96 \mathrm{kG}$. Fig.5 shows the relative energy gain as function of the square root of the laser power; the scattering of data points reflect the typical laser power pulse to pulse variation; as a consequence, every set of experimental data needs to be normalized to $\sqrt{W_{1}}$. With the present spectrometer, the energy gain could be measured with good accuracy due to the sharp intensity fall-off of the high energy edge of the non-accelerated particles. A quantitative intensity ratio of the accelerated to unaccelerated beam could not be obtained due to the extended low energy edge of the unaccelerated beam. This limited the abilityto measure the bucket size and leakage for comparison with model predictions and therefore, the value of the synchronous phase angle $\psi_{r}$ could not be unambiguously established. Analytically, $\psi_{r}$ and $\Delta \gamma / \gamma$ as a function of laser power $W_{1}$ and wiggler parameters are given by:

$$
\begin{aligned}
& \sin \psi_{r}=(3 / 16)\left(\mathrm{k} / \mathrm{k}_{\mathrm{w}}\right) \mathrm{K}\left(\mathrm{Af}(\mathrm{K}) \mathrm{L}_{\mathrm{w}}\right)^{-1}\left[\left(\lambda_{w}(\mathrm{~L}) / \lambda_{w}(0)\right)^{2}-1\right] \\
& \Delta \gamma / \gamma=2\left[\mathrm{Kf}(\mathrm{K})(\mathrm{A} / \mathrm{k})\left(1+\mathrm{K}^{2} / 2\right)^{-1}\right]^{1 / 2} \Gamma\left(\psi_{\mathrm{r}}\right)
\end{aligned}
$$

Eq.4 permits to calculate the moving bucket [12] parameter $\Gamma\left(\psi_{\mathrm{r}}\right)$ and its maximum energy extent $\Delta \gamma / \gamma$. For the experimental value $\Delta \gamma / \gamma=2.5 \%$, we find : $\psi_{\mathrm{s}}=34^{\circ}$ in reasonable agreement with the optimal $45^{\circ}$ and a laser power of $W_{1}=$ $2.7 \mathrm{GW}$ which is larger than the $1 \mathrm{GW}$ estimated experimentally.

In conclusion, the IFEL acceleration of a $40 \mathrm{MeV}$ electron beam by $\triangle \mathrm{E} / \mathrm{E}=2.5 \%$ with a $1 \mathrm{GW} \mathrm{CO}$ laser and a tapered wiggler with peak field on axis of $10 \mathrm{kG}$ has been confirmed. Agreement with the model predictions is satisfactory, permitting the scaling of anticipated results to higher laser power.

Present IFEL operation is limited to a maximum laser power of $\leq 2 \mathrm{GW}$. With the enhanced vacuum pump-out capability of the IFEL interaction region and modified entry cone to the sapphire guide configuration, as presently incorporated, operation at a laser power of $5 \mathrm{GW}$ is anticipated in the near term, which would enable close to $10 \%$ energy gain. With the upgrading of the ATF $\mathrm{CO}_{2}$ laser to the $1 \mathrm{TW}$ level as presently underway, an IFEL mean acceleration gradient of $100 \mathrm{MeV} / \mathrm{m}$ might become achievable. With regards to this, limitations on energy gain may arise from at least two sources [15]: firstly, the damage threshold of the sapphire waveguide and the consequent potential decrease of the power transmission; secondly, the decrease of the electron beam intensity associated with the self-field interaction due to the small aperture of the guide. It is intended to address these problems in future experimental work.

Near term further development of the IFEL accelerator concept will incorporate two approaches: first, the construction of a second VaP fast excitation wiggler - sapphire guide IFEL interaction region, for incorporation into a two accelerator modules IFEL accelerator, to test realistically a synchronized multi-module IFEL accelerator sequence and aim, with the above cited $\mathrm{CO}_{2}$ laser developments, at a $100 \mathrm{MeV}$ IFEL Linac ; second, in a joint developmental approach with the STI Inverse Cherenkov Accelerator (ICA) experiment [16], use of the IFEL accelerator as a synchronized prebuncher for the IC accelerator in an IFEL - ICA buncher - accelerator sequence.

The authors wish to acknowledge the continued support of I.Ben-Zvi and the technical staff of the ATF. This work was supported by the Advanced Technology R\&D Branch, Division of High Energy Physics, U.S.Department of Energy, DEAC02-76CH00016.

\section{References}

[1] R. Palmer, J. Appl. Phys. 43, $3014,1972$.

[2] C. Pellegrini, P. Sprangle,W. Zakowicz, Proc. of the XIIInt.Conf. on High EnergyAccelerators, p.473,1983.

[3] E. Courant,C. Pellegrini,W. Zakowicz, PR A32, 2813,1985.

[4] A. Fisher, J. Gallardo, J. Sandweiss, A.van Steenbergen, "Inverse Free Electron Laser Accelerator", Proc. Adv. Accel. Concepts, Port Jefferson, NY, AIP 279, p.299,1993.

[5] A. Fisher, J. Gallardo, A. van Steenbergen, J. Sandweiss, "IFEL Accelerator Development", Nucl. Instr. Meth.A341,1994

[6] I. Wernick and T. C. Marshall, Phys. Rev. A46, $3566,1992$.

[7]A.vanSteenbergen,J.Gallardo,J.Sandweiss,J.Fang,M.Babzien, K.Batchelor,A.Fisher,K.Kusche,R.Malone,L.Pogorelsky, J.Qiu, T.Romano, J.Sheehan, J.Skaritka, T.Srinivasan-Rao, XJWang, "Inverse Free Electron Laser Single Module $\mathrm{e}^{\text {" }}$ Acceleration", Proceedings BNL CAP/ATF Users Meeting, Dec., 1995

[8]A.van Steenbergen,Patent Application 368618, June '89 (Issued Aug.'90) 
[9] A. van Steenbergen, J. Gallardo, T. Romano, M. Woodle, "Fast Excitation Wiggler",Proc.PAC SF.,IEEE NS, May 1991

[10]A.Fisher,J.Gallardo,A.van Steenbergen,J.Sandweiss,J.Fang, “IFEL Development”, Mtg. Adv. Accel.,Fontana, WI '94

[11] J. Harrington, C. Gregory, Optics Letters 15, (1990)

[12] N.Kroll, P.Morton, M.Rosenbluth,Physics of QE 7,89,1979

[13] I. Ben-Zvi, Proc. Adv. Accel. Concepts, Port Jefferson, NY, AIP 279, 590 (1993)

[14] I. Pogorelsky, Proc. Adv. Accel. Concepts, Port Jefferson, NY, AIP 279608 (1993)

[15] P.Sprangle, E.Esarey , J.Krall, Phys.Plasmas 3, 2183 (1996)

[16] W.Kimura, I.Pogorelsky, Y.Liu, K.Kusche, A.van Steenbergen, J.Gallardo, J.Sandweiss, D.Quimby, M.Babzien "Inverse Cerenkov Acceleration using an IFEL Prebuncher". Proceedings this Workshop, pg .....

\begin{tabular}{|c|c|c|c|}
\hline \multirow[t]{9}{*}{$e^{-}$beam } & Injection Energy & 40.0 & $\mathrm{MeV}$ \\
\hline & Exit Energy & 42.3 & $\mathrm{MeV}$ \\
\hline & <Accel.Field> & 4.9 & $\mathrm{MV} / \mathrm{m}$ \\
\hline & Current, nominal & 5 & $\mathrm{~mA}$ \\
\hline & N(bunch) & $10^{9}$ & $e^{-}$ \\
\hline & $I(\max )$. & 30 & A \\
\hline & $\Delta \mathrm{E} / \mathrm{E}($ one $\sigma)$ & $\pm 3.10^{-3}$ & \\
\hline & Emittance (one $\sigma$ ) & $710^{-8}$ & m.rad \\
\hline & Beam radius & 0.3 & $\mathrm{~mm}$ \\
\hline \multirow[t]{6}{*}{ Wiggler } & Wiggler Length & 0.47 & $\mathfrak{m}$ \\
\hline & Section Length & 0.6 & $\mathbf{m}$ \\
\hline & Period Length, $\lambda_{w}$ & $2.9--3.1$ & $\mathrm{~cm}$ \\
\hline & Wiggler Gap & 4 & $\mathrm{~mm}$ \\
\hline & Field max. & 10 & kG \\
\hline & Beam oscill., $\mathrm{a}_{1 / 2}$ & $0.16-0.2$ & $\mathrm{~mm}$ \\
\hline \multirow[t]{8}{*}{$\mathrm{CO}_{2}$ Laser } & Power, $W_{1}$ (Laser) & $10^{9}$ & Watts \\
\hline & Wave Length, $\lambda$ & 10.6 & $\mu \mathrm{m}$ \\
\hline & Max.Field, $E_{0}$ & $0.7810^{3}$ & $\mathrm{MV} / \mathrm{m}$ \\
\hline & Guide Loss, $\alpha$ & 0.05 & $\mathrm{~m}^{-1}$ \\
\hline & Field Attenuation & 0.26 & $\mathrm{~dB} / \mathrm{Sect}$ \\
\hline & Pulse, (fwhm) & 220 & psec \\
\hline & $A_{0}$ & $1.5310^{3}$ & \\
\hline & $r_{0}\left(L_{w} / 2\right)$ & 1.0 & $\mathrm{~mm}$ \\
\hline
\end{tabular}

Sapphire Circular

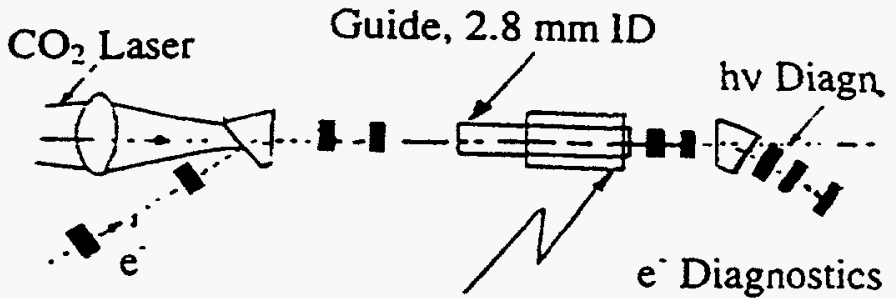

Fast Excitation

Variable Period Wiggler

FIG.1, Schematic of the experimental configuration 


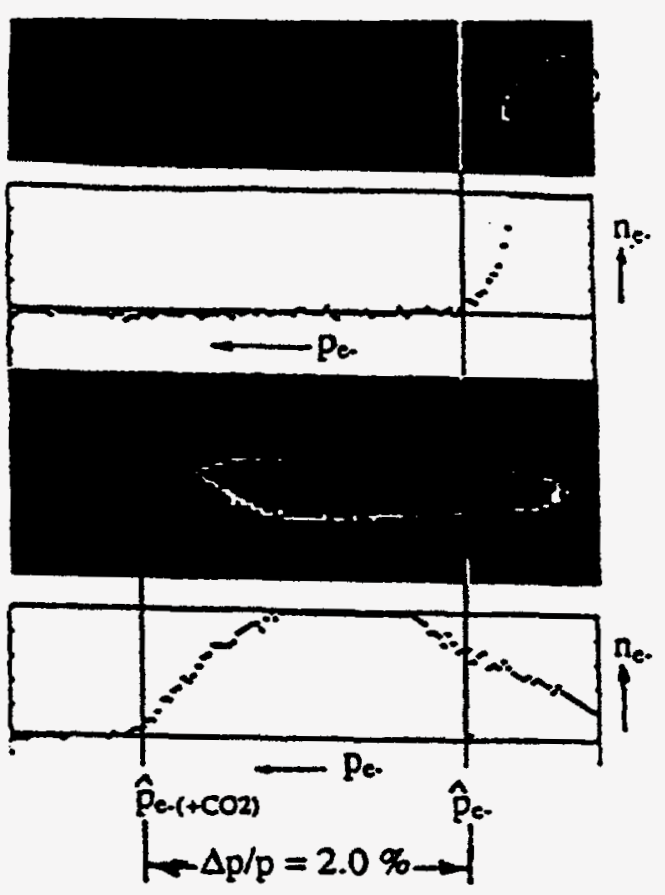

FIG. 2. Momentum spectrum of the unaccelerated and IFEL accelerated electron beam.

$\mathrm{E}_{\mathrm{l}}=40 \mathrm{MeV}, \mathrm{B}_{\mathrm{w}}=10 \mathrm{kG}, \lambda_{w}=2.9-3.1 \mathrm{~cm}, \mathrm{~W}_{\mathrm{l}}=1 \mathrm{GW}$

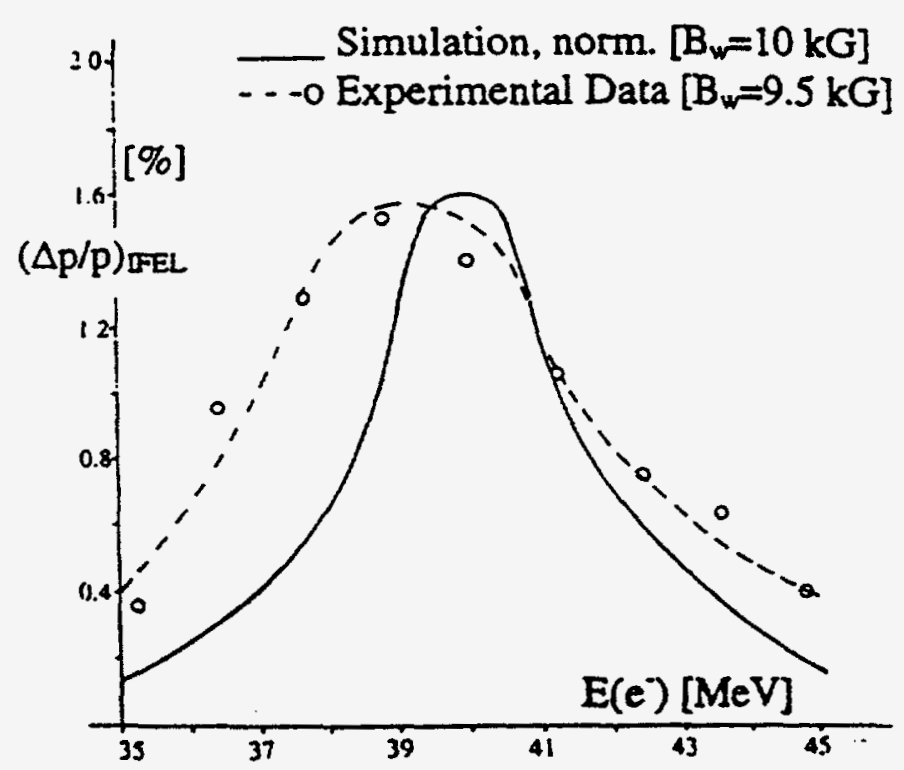

FIG. 3. Relative energy gain $\triangle E / E$ vs $E$ with $B_{w}, W_{1}$ constant
Simulation, norm.

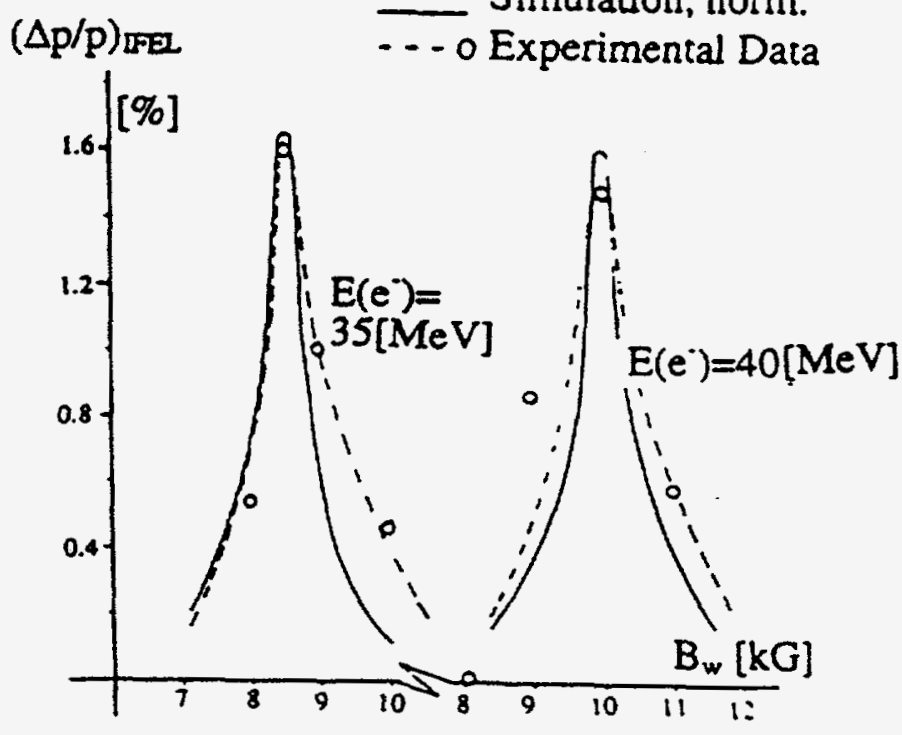

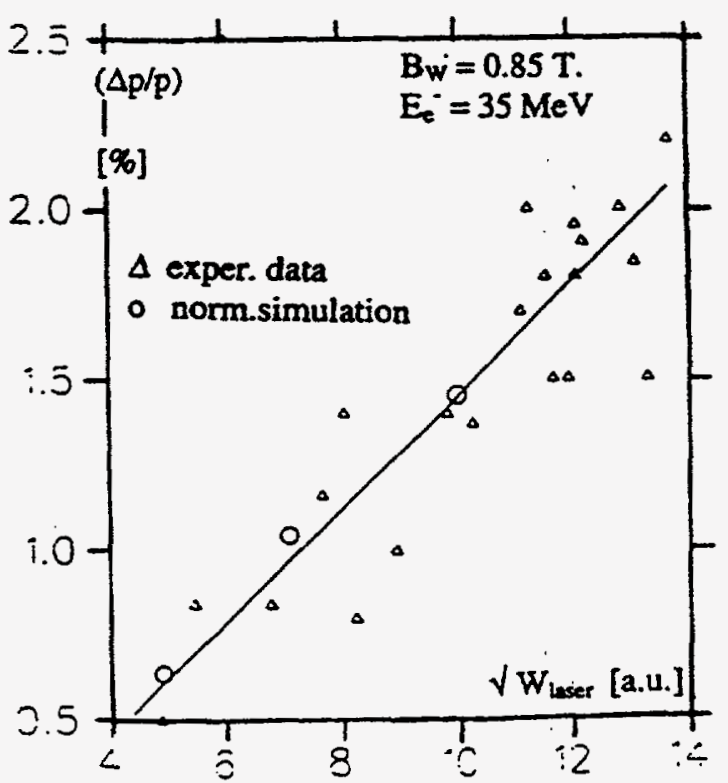

FIG. 5. Relative energy gain vs $W_{1}$ with $E$ and $B_{w}$ constant 